\title{
Tempo de armazenamento e métodos de superação da dormência de pirê- nios de Byrsonima crassifolia (L.) Kunt
}

\author{
Hygor Gomes de Almeida Sousa ${ }^{a^{*}}\left(\mathbb{D}\right.$, Bruno Aurélio Campos Aguiar ${ }^{\mathrm{a}}{ }^{\mathbb{D}}$, \\ Maristela Lima Figueiredo Guimarães Epifânio ${ }^{\mathrm{a}} \mathbb{D}$, Renata Carvalho da Silva ${ }^{\mathrm{a}} \mathbb{D}$, Priscila Bezerra de Souza ${ }^{\mathrm{a}}$ \\ ${ }^{a}$ Universidade Federal do Tocantins (UFT), Brasil \\ *Autor correspondente (hygoralmeida.floresta@outlook.com)
}

\section{N F O}

\section{Keyworks} germination treatment dormancy

\section{Palavras-chaves}

germinação tratamentos vigor

\begin{abstract}
A B S T R A C T
A methodology to evaluate the management of tourism in protected natural areas Storage time and methods of determination of pyrenes of Byrsonima crassifolia (L.) Kunt

The objective of this study was to evaluate the germination of the Byrsonima crassifolia spruces considering storage periods and dormancy overcoming methods. It was used a completely randomized design with 60 pyrenes per treatment, distributed in four replicates and eight treatments, seeded in plastic bags with dimensions of $17 \times 22 \times 0.15 \mathrm{~cm}$ containing soil from the site of the matrices and daily watering was performed to maintain substrate moisture. The storage of the pyrenes was $0,60,120$ and 180 days and the treatments totaled in 8 with control, and others carried out the chemical and mechanical superstructures. The results obtained allowed to conclude that the Byrsonima crassifolia pyrenes presented better physiological quality when stored for 120 days. Soaking in $\left(\mathrm{H}_{2} \mathrm{SO}_{4}\right)$ for 40 minutes and $\left(\mathrm{GA}_{3}\right)$ for 48 hours favored germination and provided higher rates of germination (IVG) in relation to the others.
\end{abstract}

\section{R E S U M O}

Objetivou-se avaliar a germinação dos pirênios de Byrsonima crassifolia considerando períodos de armazenamento e métodos de superação de dormência. Utilizou-se delineamento inteiramente casualizado com 60 pirênios por tratamento, distribuídos em quatro repetições e oito tratamentos, semeados em sacos plásticos com dimensões de 17 × 22 × $0,15 \mathrm{~cm}$ contendo solo do local das matrizes sendo realizadas regas diárias para manutenção da umidade do substrato. $\mathrm{O}$ armazenamento dos pirênios foi de $0,60,120$ e 180 dias e os tratamentos totalizaram em 8 com testemunha, e outros realizado as superações química e mecânica. Os resultados obtidos permitiram concluir que os pirênios de Byrsonima crassifolia apresentaram melhor qualidade fisiológica quando armazenadas por 120 dias. A embebição em $\left(\mathrm{H}_{2} \mathrm{SO}_{4}\right)$ por 40 minutos e $\left(\mathrm{GA}_{3}\right)$ por 48 horas favoreceram a germinação e proporcionaram maiores índices de velocidade de germinação (IVG) em relação aos demais. 


\section{INTRODUÇÃO}

Os pirênios da maioria das espécies florestais germinam prontamente quando lhes são dadas condições ambientais favoráveis. No entanto cerca de dois terços das espécies arbóreas apresentam certo grau de dormência, que pode ser superada com a utilização de tratamentos pré-germinativos (Oliveira et al., 2012).

Na maioria das espécies florestais, a dormência de pirênios é um fato comum, sendo esta, em condições naturais, de grande valor por ser um mecanismo de sobrevivência da espécie. No entanto, passa a ser um problema quando os pirênios são utilizadas para a produção de mudas em razão do longo tempo necessário para a germinação, ficando as mesmas sujeitas a condições adversas (Borges et al.,1982; Melo et al., 2011).

Entre os tratamentos utilizados com sucesso para a superação da dormência tegumentar de espécies florestais, destacam-se as escarificações mecânica e química, além da imersão dos pirênios em água quente (Scalon et al., 2005).

Os pirênios do gênero Byrsonima apresentam dormência devido à impermeabilidade do tegumento, como já foi observado por Vasconcelos Filho (2008), além disso, diversos métodos para superação de dormência de murici vêm sendo empregados por produtores e pesquisadores para obter maior e melhor uniformidade e aceleração da germinação dos pirênios, entretanto, os estudos ainda são escassos e sem nenhuma eficiência comprovada. Visto que a propagação através de pirênios do gênero Byrsonima esbarra em problemas como baixa taxa de germinação e emergência lenta das plântulas, sendo isso decorrente de presença de um endocarpo esclerificado que envolve o embrião e que atua como barreira mecânica. Em condições naturais ou de viveiro, a germinação do murici é baixa, irregular e lenta, o que tem inviabilizado a produção de mudas deste gênero (Morais Júnior et al., 2015).

Dessa forma, conhecer os mecanismos de dormência, duração e métodos de facilitar a germinação de algumas sementes de espécies florestais tem importância tanto ecológica, quanto econômica (Murakami et al., 2011).

Diante do contexto, objetivou-se avaliar a germinação dos pirênios de Byrsonima crassifolia (L.) Kunt considerando períodos de armazenamento e métodos de superação de dormência.

\section{MATERIALES E MÉTODOS}

O experimento foi conduzido no TERRAQUARIUM, fragmento de Cerrado do Centro Universi- tário Luterano de Palmas - CEULP/ULBRA, Palmas (TO), com coordenada $10^{\circ} 16^{\prime} 96^{\prime \prime} \mathrm{S}$ e $48^{\circ} 20^{\prime} 03^{\prime}$ ' W. O clima da região é marcadamente estacional com duas estações bem definidas, com cerca de seis meses de seca compreendendo o período de inverno e seis meses de chuva que correspondem ao verão.

Os frutos de Byrsonima crassifolia (L.) Kunt. foram coletados aleatoriamente em 40 matrizes de um fragmento de Cerrado sensu stricto inserido no Reassentamento Mariana, localizado à $17 \mathrm{~km}$ do município de Palmas (TO), após a coleta dos frutos os mesmos foram transportados para o Laboratório de Sementes Ulbra, Palmas (TO) onde prosseguiu para o beneficiamento, secagem e armazenamento.

Empregou-se um delineamento experimental inteiramente casualizado com 60 pirênios por tratamento, distribuídos em quatro repetições, sendo as análises realizadas para o $0,60,120$ e 180 dias de armazenamento. Foram testados os seguintes tratamentos pré-germinativos com o intuito de antecipar, aumentar e uniformizar a porcentagem de germinação dos pirênios: $\mathrm{T}_{1}=$ Testemunha; $\mathrm{T}_{2}=$ Escarificação química com ácido sulfúrico $\left(\mathrm{H}_{2} \mathrm{SO}_{4}\right)$ concentrado por 20 minutos; $\mathrm{T}_{3}=$ Escarificação química com ácido sulfúrico $\left(\mathrm{H}_{2} \mathrm{SO}_{4}\right)$ concentrado por 40 minutos; $\mathrm{T}_{4}=$. Escarificação química com ácido giberélico na concentração $500 \mathrm{mg} \mathrm{L}^{-1}\left(\mathrm{GA}_{3}\right)$ por 24 horas; $\mathrm{T}_{5}=$. Escarificação química com ácido giberélico na concentração $500 \mathrm{mg} \mathrm{L}^{-1}\left(\mathrm{GA}_{3}\right)$ por 48 horas; $\mathrm{T}_{6}=$ Escarificação mecânica com lixa número 80 por 30 segundos; $T_{7}=$ Escarificação mecânica com lixa número 80 por 30 segundos mais imersão em água por 24 horas; $\mathrm{T}_{8}=$ Escarificação mecânica com lixa número 80 por 30 segundos mais imersão em água por 48 horas.

A escarificação mecânica com lixa foi efetuada friccionando individualmente cada pirênio de forma padronizada do local de atrito, o pecíolo, mas de forma superficial por 30 segundos. Os pirênios de cada tratamento descrito anteriormente foram submetidos ao teste de germinação e a semeadura foi realizada em sacos plásticos com dimensões de $17 \times 22 \times 0,15 \mathrm{~cm}$ contendo solo do local das matrizes sendo realizadas regas diárias para manutenção da umidade do substrato.

O experimento constituído por pirênios (ponto zero), ou seja, sem nenhum dia de armazenamento foram submetidos aos tratamentos $\left(\mathrm{T}_{1}\right.$ ao $\left.\mathrm{T}_{8}\right)$ sequencialmente foram semeados em sacos plásticos com dimensões de 17 × 22 × $0,15 \mathrm{~cm}$ contendo solo do local das matrizes sendo realizadas regas diárias para manutenção da umidade do substrato, sendo os tratamentos realizados para os pirênios armazenados por 60,120 e 180 dias.

O período de observação da germinação foi de 180 dias, sendo feita a contagem diária dos pirênios 
germinados, dessa forma foram avaliados a porcentagem de germinação $\left(\mathrm{G}_{(\%)}\right)$ e o índice de velocidade de germinação (IVG) de acordo com a fórmula proposta por Maguire (1962).

$$
\begin{aligned}
& G_{(\%)}=\left(\frac{P n}{T S}\right) 100 \\
& I V G=\frac{G 1}{N 1}+\frac{G 2}{N 2}+\cdots \cdots+\frac{G i}{N i}
\end{aligned}
$$

Em que: $\mathrm{Gi}$ = número de plântulas computadas na primeira, segunda, terceira e última contagem; $\mathrm{Ni}=$ número de dias da semeadora à primeira, segunda, terceira e última contagem; $\mathrm{Pn}=$ plântulas normais germinadas; $\mathrm{TS}=$ número total de pirênios colocadas para germinar.

Os dados de porcentagem de germinação foram submetidos ao teste de normalidade de ShapiroWilk(W) não havendo necessidade de transformação. Sendo posteriormente submetidos à análise de variância (ANOVA) e as médias comparadas pelo teste Tukey, ao nível de 5\% de probabilidade.

\section{RESULTADOS E DISCUSSÃO}

Os pirênios considerado ponto-zero, ou seja, pirênios que não foram armazenados obtiveram baixo índice de germinação, entretanto o tratamento $T_{4}$ com imersão em $\mathrm{GA}_{3}$ por 24 horas obtiveram maior porcentagem de germinação de $11,62 \%$, os tratamentos $\mathrm{T}_{1}$ Testemunha e $\mathrm{T}_{7}$ escarificação com Lixa $+\mathrm{H}_{2} \mathrm{O}$ por 24 horas obtiveram resultados não significativos na porcentagem de germinação por não terem nenhum pirênio germinado ao longo do tempo de análise. Já o $\mathrm{T}_{2} \mathrm{H}_{2} \mathrm{SO}_{4}$ por 20 minutos e o $\mathrm{T}_{8} \mathrm{Lixa}+\mathrm{H}_{2} \mathrm{O}$ por 48 horas, obtiveram porcentagem de germinação de $1,65 \%$ e $\mathrm{T}_{6}$ Lixa obteve porcentagem de germinação de $3,3 \%$, diferente do $\mathrm{T}_{3}$ $\mathrm{H}_{2} \mathrm{SO}_{4}$ por 40 minutos e $\mathrm{T}_{5} \mathrm{GA}_{3}$ por 48 horas que obtiveram quantidade mais significativa na porcentagem de germinação sendo 6,65\% e 9,95\% respectivamente, dessa forma pode-se inferir que os tratamentos $\mathrm{T}_{3}, \mathrm{~T}_{4}$ e $\mathrm{T}_{5}$ proporcionaram melhores resultados, além de serem estatisticamente iguais a 5\% de probabilidade pelo teste de Tukey (Tabela 01).

Os tratamentos $\mathrm{T}_{7}$ e $\mathrm{T}_{8}$ com lixiviação e imersão em água por 24 e 48 horas respectivamente em Byrsonima crassifolia (L.) Kunt obtiveram os menores índices de germinação, contudo testando o tempo de armazenamento ponto-zero o resultado foi negativo não havendo germinação em ambos os tempos analisados (Tabela 01).
Tabela 01 - Porcentagem de germinação $(\mathrm{G} \%)$ e índice de velocidade de germinação (IVG) dos pirênios de Byrsonima crassifolia (L.) Kunt. pontozero (sem armazenamento) submetido a diferentes tratamentos de superação da dormência.

\begin{tabular}{lrl}
\hline \multicolumn{1}{c}{ Tratamentos } & \multicolumn{1}{c}{ G\% } & IVG \\
\hline $\mathrm{T}_{1}-$ Testemunha & $0,0000 \mathrm{c}$ & $0,0000 \mathrm{~b}$ \\
$\mathrm{~T}_{2}-\mathrm{H}_{2} \mathrm{SO}_{4} 20 \mathrm{~min}$ & $1,6500 \mathrm{c}$ & $0,0014 \mathrm{~b}$ \\
$\mathrm{~T}_{3}-\mathrm{H}_{2} \mathrm{SO}_{4} 40 \mathrm{~min}$ & $6,6500 \mathrm{abc}$ & $0,0111 \mathrm{~b}$ \\
$\mathrm{~T}_{4}-\mathrm{GA}_{3} 24 \mathrm{H}$ & $11,6200 \mathrm{a}$ & $0,0432 \mathrm{a}$ \\
$\mathrm{T}_{5}-\mathrm{GA}_{3} 48 \mathrm{H}$ & $9,9500 \mathrm{ab}$ & $0,0395 \mathrm{a}$ \\
$\mathrm{T}_{6}-\mathrm{Lixa}$ & $3,3000 \mathrm{bc}$ & $0,0044 \mathrm{~b}$ \\
$\mathrm{~T}_{7}-\mathrm{Lixa}+\mathrm{H}_{2} \mathrm{O} 24 \mathrm{~h}$ & $0,0000 \mathrm{c}$ & $0,0000 \mathrm{~b}$ \\
$\mathrm{~T}_{8}-\mathrm{Lixa}+\mathrm{H}_{2} \mathrm{O} 48 \mathrm{~h}$ & $1,6500 \mathrm{c}$ & $0,0017 \mathrm{~b}$ \\
\hline
\end{tabular}

** As médias seguidas pela mesma letra não diferem estatisticamente entre si. Foi aplicado o teste Tukey ao nível de 5\% de probabilidade.

De acordo com Murakami et al. (2011), a germinação da espécie Byrsonima cydoniifolia A. Juss. deve ser realizada com pirênios obtidas de frutos maduros, que sofreram abscisão natural, utilizando pirênios com endocarpos íntegros embebidos em ácido giberélico $\mathrm{GA}_{3}$ na concentração de $1 \mathrm{~g} \mathrm{~L}^{-1}$ por 24 horas. Os resultados obtidos por Murakami et al. (2011) e Morais Júnior et al. (2015) utilizando Byrsonima sp. corroboram com o presente trabalho, visto que o $\mathrm{GA}_{3}$ em ambos tempos de embebição 24 horas e 48 horas apresentaram-se valores satisfatórios, o que demonstra ser uma técnica de superação de dormência superior aos demais tratamentos testados.

Segundo Lopes et al. (1998), os mesmos não obtiveram resultados positivos na embebição por 48 horas em água com pirênios de Samanea saman (Jacq.) Merr., em contrapartida nesse estudo com pirênios de Byrsonima crassifolia (L.) Kunt embebidos em água após escarificação com lixa obtiveram resultados satisfatórios.

Ao trabalharem com Byrsonima crassifolia (L.) Kunt, Nascimento et al. (2009) utilizaram $\mathrm{H}_{2} \mathrm{SO}_{4}$ para a superação da dormência da espécie descrita acima, obtiveram resultados inferiores, segundo o referido autor todos os tempos de imersão no ácido sulfúrico foram prejudiciais ao desenvolvimento do eixo embrionário causando possivelmente a morte do embrião ou algum dano aos mecanismos responsáveis pelo processo de germinação. Dados estes que corroboram com os resultados do presente estudo pirênios de Byrsonima crassifolia (L.) Kunt sem nenhum dia de armazenamento (ponto-zero), pois obteve-se baixos valores no índice de velocidade de germinação sendo de 0,0050 em $\mathrm{T}_{2} \mathrm{H}_{2} \mathrm{SO}_{4}$ por 20 minutos (Tabela 01).

Cabe ressaltar que os pirênios de Byrsonima crassifolia (L.) Kunt (ponto-zero) não obtiveram 
resultados positivos para testemunha, entretanto obteve-se resultados positivos para a germinação utilizando $\mathrm{GA}_{3}$ a $0,60,120$ e 180 dias de armazenamento.

A germinação dos pirênios de Byrsonima crassifolia (L.) Kunt (testemunha) sem nenhum tratamento iniciou-se a partir dos 60 dias de armazenamento. Após 60 dias de armazenamento os pirênios de Byrsonima crassifolia (L.) Kunt foram submetidos aos oito tratamentos de superação de dormência e sequencialmente foram semeados em sacos plásticos. A maior porcentagem de germinação foi para $\mathrm{T}_{3}$ imersos em $\mathrm{H}_{2} \mathrm{SO}_{4}$ por 40 minutos e $\mathrm{T}_{6}$ Lixa sendo de $9,97 \%$. Em seguida $\mathrm{T}_{6}$ e $\mathrm{T}_{7}$ escarificação com Lixa e Lixa $+\mathrm{H}_{2} \mathrm{O}$ por 24 horas ambos apresentaram mesma porcentagem de germinação de $10 \%$. Vale ressaltar que apenas em $\mathrm{T}_{8}$ escarificação com Lixa $+\mathrm{H}_{2} \mathrm{O}$ por 48 horas não apresentou resultados satisfatórios na germinação dos pirênios (Tabela 02). Para Nascimento et. al. (2009) a escarificação com lixa e escarificação com lixa + imersão em água destilada por 24 horas, não diferiram estatisticamente do tratamento $\mathrm{T}_{1}$ testemunha.

O IVG dos tratamentos $T_{2}$ escarificação química $\mathrm{H}_{2} \mathrm{SO}_{4}$ por 20 minutos e $\mathrm{T}_{6}$ escarificação mecânica com Lixa obtiveram maiores valores, resultado que difere do $\mathrm{T}_{8}$ escarificação com Lixa $+\mathrm{H}_{2} \mathrm{O}$ por 48 horas onde o mesmo não foi satisfatório, já o tratamento $T_{1}$ obteve baixo IVG, porém significativo quando comparado ao $\mathrm{T}_{8}$ (Tabela 02).

Tabela 02 - Porcentagem de germinação $(\mathrm{G} \%)$ e índice de velocidade de germinação (IVG) dos pirênios de Byrsonima crassifolia (L.) Kunt com 60 dias de armazenamento e submetidos a diferentes tratamentos de superação de dormência.

\begin{tabular}{lll}
\hline \multicolumn{1}{c}{ Tratamentos } & \multicolumn{1}{c}{ G $\%$} & \multicolumn{1}{c}{ IVG } \\
\hline $\mathrm{T}_{1}-$ Testemunha & $4,9700 \mathrm{ab}$ & $0,0114 \mathrm{ab}$ \\
$\mathrm{T}_{2}-\mathrm{H}_{2} \mathrm{SO}_{4} 20 \mathrm{~min}$ & $8,3000 \mathrm{ab}$ & $0,0353 \mathrm{ab}$ \\
$\mathrm{T}_{3}-\mathrm{H}_{2} \mathrm{SO}_{4} 40 \mathrm{~min}$ & $9,9700 \mathrm{a}$ & $0,0355 \mathrm{ab}$ \\
$\mathrm{T}_{4}-\mathrm{GA}_{3} 24 \mathrm{H}$ & $6,6200 \mathrm{ab}$ & $0,0242 \mathrm{ab}$ \\
$\mathrm{T}_{5}-\mathrm{GA}_{3} 48 \mathrm{H}$ & $8,30000 \mathrm{ab}$ & $0,0323 \mathrm{ab}$ \\
$\mathrm{T}_{6}$ Lixa & $9,9700 \mathrm{a}$ & $0,0447 \mathrm{a}$ \\
$\mathrm{T}_{7}-\mathrm{Lixa}+\mathrm{H}_{2} \mathrm{O} 24 \mathrm{~h}$ & 9,9500 & $0,0208 \mathrm{ab}$ \\
$\mathrm{T}_{8}-\mathrm{Lixa}+\mathrm{H}_{2} \mathrm{O} 48 \mathrm{~h}$ & $0,0000 \mathrm{~b}$ & $0,0000 \mathrm{~b}$ \\
\hline
\end{tabular}

** As médias seguidas pela mesma letra não diferem estatisticamente entre si. Foi aplicado o teste Tukey ao nível de 5\% de probabilidade.

Conforme Scalon et al. (2006) afirmam que os pirênios de Jacaranda cuspidifolia Mart. não devem ser semeadas logo após a extração dos frutos, pois os mesmos observaram que a porcentagem de emergência aumentou com o período de armazenamento e refrigeração Dados estes que corroboram com o presente estudo, que ao compararmos a porcentagem de germinação e o índice de velocidade de germinação dos pirênios de Byrsonima crassifolia recém-colhidos (ponto-zero) para os a 120 dias de armazenamento o que pode concluir que os pirênios envelhecidos apresentaram maior vigor com relação aos recém-colhidos, considerando ainda os métodos utilizados para a superação da dormência (Tabela 01 e Tabela 03).

Os pirênios armazenados por 120 dias foram submetidos aos tratamentos e sequencialmente semeados em sacos plásticos. Aos 120 dias constatouse um aumento significativo da porcentagem de germinação, quando comparado ao tempo de armazenamento de 30 e 60 dias. Desta forma podemos inferir que pirênios de Byrsonima crassifolia armazenados por 120 dias apresentaram maior viabilidade para a germinação (Tabela 03).

Tabela 03 - Porcentagem de germinação $(\mathrm{G} \%)$ e índice de velocidade de germinação (IVG) de pirênio de Byrsonima crassifolia (L.) Kunt com 120 dias de armazenamento posteriormente submetidos a diferentes tratamentos de superação da dormência.

\begin{tabular}{lll}
\hline \multicolumn{1}{c}{ Tratamentos } & \multicolumn{1}{c}{ G\% } & \multicolumn{1}{c}{ IVG } \\
\hline $\mathrm{T}_{1}-$ Testemunha & $16,65 \mathrm{~b}$ & $0,05138 \mathrm{~b}$ \\
$\mathrm{~T}_{2}-\mathrm{H}_{2} \mathrm{SO}_{4} 20 \mathrm{~min}$ & $21,62 \mathrm{ab}$ & $0,06833 \mathrm{ab}$ \\
$\mathrm{T}_{3}-\mathrm{H}_{2} \mathrm{SO}_{4} 40 \mathrm{~min}$ & $33,30 \mathrm{ab}$ & $0,13728 \mathrm{a}$ \\
$\mathrm{T}_{4}-\mathrm{GA}_{3} 24 \mathrm{H}$ & $16,62 \mathrm{~b}$ & $0,06988 \mathrm{ab}$ \\
$\mathrm{T}_{5}-\mathrm{GA}_{3} 48 \mathrm{H}$ & $34,97 \mathrm{a}$ & $0,13700 \mathrm{a}$ \\
$\mathrm{T}_{6}-\mathrm{Lixa}$ & $21,62 \mathrm{ab}$ & $0,07275 \mathrm{ab}$ \\
$\mathrm{T}_{7}-\mathrm{Lixa}+\mathrm{H}_{2} \mathrm{O} 24 \mathrm{~h}$ & $28,30 \mathrm{ab}$ & $0,10198 \mathrm{ab}$ \\
$\mathrm{T}_{8}-$ Lixa+$+\mathrm{H}_{2} \mathrm{O} 48 \mathrm{~h}$ & $24,95 \mathrm{ab}$ & $0,08438 \mathrm{ab}$ \\
\hline
\end{tabular}

** As médias seguidas pela mesma letra não diferem estatisticamente entre si. Foi aplicado o teste Tukey ao nível de 5\% de probabilidade.

Os pirênios armazenados por 120 dias foram submetidos aos tratamentos e sequencialmente semeados em sacos plásticos. Aos 120 dias constatouse um aumento significativo da porcentagem de germinação, quando comparado ao tempo de armazenamento de 30 e 60 dias. Desta forma podemos inferir que pirênios de Byrsonima crassifolia armazenados por 120 dias apresentaram maior viabilidade para a germinação (Tabela 03).

$\mathrm{O}$ tratamento $\mathrm{T}_{5}$ imersão em $\mathrm{GA}_{3}$ por 48 horas obteve maior porcentagem de germinação de $34,97 \%$, seguido de $\mathrm{T}_{3} \mathrm{H}_{2} \mathrm{SO}_{4}$ por 40 minutos $33,3 \%, \mathrm{~T}_{7} \mathrm{Lixa}+\mathrm{H}_{2} \mathrm{O}$ por 24 horas $28,3 \%, \mathrm{~T}_{8}$ Lixa $+\mathrm{H}_{2} \mathrm{O}$ por 48 horas $24,95 \%$, já nos tratamentos $\mathrm{T}_{6}$ Lixa e $\mathrm{T}_{2} \mathrm{H}_{2} \mathrm{SO}_{4}$ por 20 minutos obtiveram mesma porcentagem de germinação de $21,6 \%$, por 
fim tratamento $\mathrm{T}_{1}$ Testemunha e $\mathrm{T}_{4} \mathrm{GA}_{3}$ por 24 horas obtiveram $16,6 \%$ de germinação (Tabela 03 ).

Segundo Biruel et al. (2007) ressaltaram que os pirênios de Caesalpinia leiostachya (Benth.) Ducke (pau-ferro) armazenadas, quando expostas a imersão em ácido sulfúrico por $10 \mathrm{~min}$ se tornam suficientes para superar a dormência causada pela impermeabilidade do tegumento à água. Já os pirênios recém-colhidas, imersas em ácido sulfúrico por 20 a $30 \mathrm{~min}$ favorece a porcentagem e a velocidade de germinação.

Em contrapartida os resultados obtidos para Byrsonima crassifolia (L.) Kunt no tratamento $\mathrm{T}_{3}$ com $\mathrm{H}_{2} \mathrm{SO}_{4}$ por 40 minutos foram satisfatórios sendo que os melhores resultados foram obtidos através dos pirênios com tempo de armazenamento $120 \mathrm{e}$ 180 dias com destaque para os 120 dias propiciando aumento da porcentagem de germinação e aumento no índice de velocidade de germinação (Tabela 3 e 4).

O armazenamento associado aos métodos de superação de dormência dos pirênios de Byrsonima crassifolia (L.) Kunt foi de grande importância para obter-se resultados satisfatórios nesse estudo (Tabela 04).

De acordo com Cabral et al. (2003) observam que a capacidade germinativa dos pirênios de $T a$ bebuia aurea (Manso), armazenadas em câmara fria e seca foram mantidas por 120 dias, ou seja, a qualidade fisiológica dos pirênios não foram afetadas pela permeabilidade das embalagens. Esses resultados concordam com os obtidos por Souza et al. (1980a e b) para outras espécies características da região semi-árida nordestina como: Anadenanthera macrocarpa (Benth.), Tabebuia impetiginosa (Mart. ex DC.), Pseudobombax simplicifolium A. Robyns e Astronium urundeuva (Fr.All) Engl., armazenadas em câmara fria $\left(8^{\circ} \mathrm{C}\right.$ e $50 \%$ UR). Os resultados destes trabalhos corroboram com os resultados do período de armazenagem dos pirênios de Byrsonima crassifolia (L.) Kunt, sendo que os 120 dias, apresentaram maiores taxas de germinação em todos tratamentos.

Os pirênios armazenados por 180 dias foram submetidos aos tratamentos e sequencialmente semeados em sacos plásticos. Observou-se que a porcentagem mais significativa foi para o tratamento $\mathrm{T}_{2} \mathrm{H}_{2} \mathrm{SO}_{4}$ por 20 minutos $16,6 \%$. Os demais tratamentos não obtiveram índices superiores e nem apresentaram grandes diferenças entre si, porém considerando o armazenamento de 180 dias, a germinação dos pirênios de Byrsonima crassifolia (L.) Kunt não foi negativa em nenhum dos métodos de superação testados (Tabela 04).

A diferença mais significativa aos 180 dias de armazenamento, referente ao IVG ocorreu nos tratamentos $\mathrm{T}_{2} \mathrm{H}_{2} \mathrm{SO}_{4}$ por 20 minutos e o $\mathrm{T}_{5} \mathrm{GA}_{3}$ por
48 horas, os menores IVG foram encontrados no Tratamento $\mathrm{T}_{1}$ Testemunha e $\mathrm{T}_{8} \mathrm{Lixa}+\mathrm{H}_{2} \mathrm{O}$ por 48 horas (Tabela 04).

Tabela 04 - Porcentagem de germinação $(\mathrm{G} \%)$ e índice de velocidade de germinação (IVG) dos pirênios de Byrsonima crassifolia (L.) Kunt. com 180 dias de armazenamento posteriormente submetidos a diferentes tratamentos de superação da dormência.

\begin{tabular}{lrr}
\multicolumn{1}{c}{ Tratamentos } & \multicolumn{1}{c}{ G\% } & \multicolumn{1}{c}{ IVG } \\
\hline $\mathrm{T}_{1}-$ Testemunha & $6,65 \mathrm{ab}$ & $0,01505 \mathrm{~b}$ \\
$\mathrm{~T}_{2}-\mathrm{H}_{2} \mathrm{SO}_{4} 20 \mathrm{~min}$ & $16,62 \mathrm{a}$ & $0,07323 \mathrm{a}$ \\
$\mathrm{T}_{3}-\mathrm{H}_{2} \mathrm{SO}_{4} 40 \mathrm{~min}$ & $11,62 \mathrm{ab}$ & $0,04768 \mathrm{ab}$ \\
$\mathrm{T}_{4}-\mathrm{GA}_{3} 24 \mathrm{H}$ & $4,95 \mathrm{ab}$ & $0,02648 \mathrm{ab}$ \\
$\mathrm{T}_{5}-\mathrm{GA}_{3} 48 \mathrm{H}$ & $13,30 \mathrm{ab}$ & $0,04330 \mathrm{ab}$ \\
$\mathrm{T}_{6} \mathrm{Lixa}$ & $4,97 \mathrm{ab}$ & $0,02015 \mathrm{~b}$ \\
$\mathrm{~T}_{7}-\mathrm{Lixa}+\mathrm{H}_{2} \mathrm{O} 24 \mathrm{~h}$ & $9,95 \mathrm{ab}$ & $0,04240 \mathrm{ab}$ \\
$\mathrm{T}_{8}-\mathrm{Lixa}+\mathrm{H}_{2} \mathrm{O} 48 \mathrm{~h}$ & $3,30 \mathrm{~b}$ & $0,01520 \mathrm{~b}$ \\
\hline
\end{tabular}

** As médias seguidas pela mesma letra não diferem estatisticamente entre si. Foi aplicado o teste Tukey ao nível de 5\% de probabilidade.

No presente estudo a concentração de $\mathrm{GA}_{3}$ utilizada foi $500 \mathrm{mg} \mathrm{L}^{-1}$, com imersão por 24 e 48 horas respectivamente nos pirênios de Byrsonima crassifolia (L.) Kunt, observou-se que mesmo sem o trincamento do endorcarpo, mantendo-os totalmente íntegros o $\mathrm{GA}_{3}$ foi absorvido, o que influenciou no desenvolvimento do embrião e consequentemente na germinação dos pirênios. Dentre os oito tratamentos de superação de dormência testados o $\mathrm{GA}_{3}$ está entre o mais satisfatório na germinação de $B y r$ sonima crassifolia (L.) Kunt.

De acordo com Passos et al. (2004) o GA3, promovem a germinação de pirênios em várias espécies. Passos et al. (2004) propôs que o $\mathrm{GA}_{3}$ promove a germinação de pirênios estimulando o crescimento do embrião e induzindo a produção de hidrolases para enfraquecer as estruturas ao redor do embrião.

$\mathrm{O}$ tratamento $\mathrm{T}_{3} \mathrm{H}_{2} \mathrm{SO}_{4}$ concentrado, obteve resultados positivos com pirênios totalmente íntegros o tempo de imersão por 40 minutos teve-se uma porcentagem de germinação superior ao de $20 \mathrm{mi}-$ nutos, porém ambos os tratamentos foram relevantes para a germinação de Byrsonima crassifolia $(\mathrm{L}$.) Kunt. Os resultados corroboram com os obtidos por Martins et al. (1992) que trabalharam com pirênios de Mimosa caesalpiniaefolia Benth. onde recomendaram a imersão em ácido sulfúrico $(95 \%)$ com tempo variado, por proporcionar as maiores porcentagens de germinação. Já Loureiro et al. (1995) 
constataram que a imersão dos pirênios de Bowdichia virgilioides Kunth (sucupira-preta) em ácido sulfúrico, com diferentes períodos foram os tratamentos mais efetivos para germinação dessa espécie.

Os resultados com pirênios de Byrsonima crassifolia (L.) Kunt no presente estudo não foram superiores a 50\% mesmo com diferentes períodos de armazenamento. Na figura 01, pode-se visualizar as porcentagens média dos tratamentos em diferentes períodos de armazenamento, podendo concluir que em todos os tratamentos a melhor época de armazenamento foi aos 120 dias, apresentando maior emergência nos diferentes tratamentos.

Figura 01 - Porcentagem média de germinação

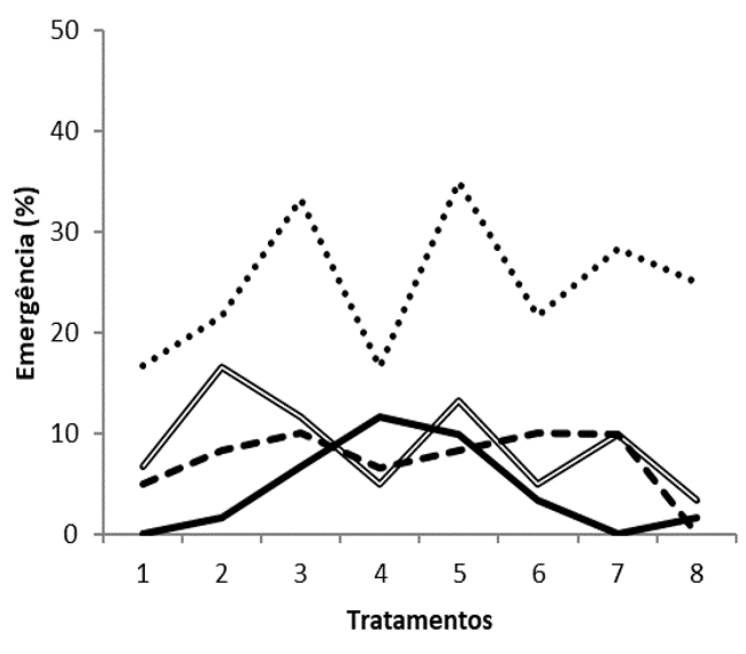

-0dias $\quad-$-60dias....$\cdot 120$ dias $\longrightarrow$ 180dias

$(\mathrm{G} \%)$ dos quatro tempos de armazenamento dos pirênios de Byrsonima crassifolia (L.) Kunt, na testemunha e nos tratamentos químicos e mecânicos testados.

Foi observado que para o ponto-zero (sem nenhum dia de armazenamento) o tratamento que se destacou foi o $\mathrm{T} 4$ de $\mathrm{GA}_{3}$ por 24 horas, já para 60 dias de armazenamento o uso da Lixa nos tratamentos obteve melhores índices de emergência. Aos 120 dias de armazenamento o uso de $\mathrm{GA}_{3}$ por 48 horas e $\mathrm{H}_{2} \mathrm{SO}_{4}$ por 40 minutos, demostraram melhores resultados, já para 180 de armazenamento o melhor resultado foi para imersão em $\mathrm{H}_{2} \mathrm{SO}_{4}$ por 20 minutos.

Conforme Scalon et al. (2005) observaram que pirênios de Enterolobium contortisiliquum (Vell.) Morong apresentaram elevada porcentagem de germinação e IVG quando escarificadas; entretanto, foi observada redução no potencial germinativo durante o armazenamento. Em contrapartida, neste estudo os pirênios de Byrsonima crassifolia (L.) Kunt obtiveram melhores resultados na germinação após o armazenamento.

\section{CONCLUSÕES}

Dessa forma, pode-se concluir que a germinação dos pirênios de Byrsonima crassifolia (L.) Kunt. aumentou com o período de armazenamento aos 120 dias, associado aos métodos de escarificação química com $\mathrm{H}_{2} \mathrm{SO}_{4}$ por 40 minutos e $\mathrm{GA}_{3}$ por 48 horas, pois apresentaram maior porcentagem de germinação em relação ao ponto-zero (sem nenhum dia de armazenamento) e 60 dias de armazenamento.

A associação do armazenamento com os métodos de superação da dormência possibilitou um aumento gradativo da germinação dos pirênios de Byrsonima crassifolia (L.) Kunt, entretanto a porcentagem de germinação e índice de velocidade de germinação permaneceram baixos quando comparados a outras espécies florestais.

\section{REFERÊNCIAS BIBLIOGRÁFICAS}

Biruel RP, Aguiar IB, Paula RC. Germinação de sementes de pau-ferro submetidas a diferentes condições de armazenamento, escarificação química, temperatura e luz. Revista Brasileira de Sementes, v.29, n.3, p.151-159, 2007.

Borges EEL, Borges RCG, Candido JF, Gomes JM. Comparação de métodos de quebra de dormência em sementes de copaíba. Revista Brasileira de Sementes, v.4, n.1, p.9-12, 1982.

Cabral EL, Barbosa DCA, Simabukuro EA. Armazenamento e germinação de sementes de Tabebuia aurea (Manso) Benth. \& Hook. F. EX. S. Moore. Acta Botânica Brasileira, Recife, v.17, n.4, p.609-617, 2003.

Lopes JC, Capucho MT, Krohling B, Zanotti P. Germinação de sementes de espécies florestais de Caesalpinia ferrea Mart. ex Tul. var. Leiostachya Benth., Cassia grandis L. e Samanea saman MERRILL, após tratamentos para superar a dormência. Revista Brasileira de Sementes, Brasília, v.20, n.1, p.80-86, 1998.

Loureiro MB, Andrade ACS, Ramos FN, Souza ADO. Quebra de dormência de sementes de sucupira-preta (Bowdichia virgilioides H.B.K. In: Congresso brasileiro de sementes, IX, Florianópolis-SC, ago. 23-27. Informativo ABRATES, Londrina, v.5, n.2, p.202. 1995.

Maguire JD. 1962. Speed of germination-aid in selection and evaluation for seedling emergence and vigor. Crop Science, v.1, p.176-177, 1962.

Martins R, Carvalho NM, Oliveira AP. Quebra de dormência de sementes de sabiá (Mimosa caesalpiniaefolia BENTH.). Revista Brasileira de Sementes, Brasília, v.14, n.1, p.5-8. 1992.

Melo MGG, Mendonça MS, Nazário PM, Angela MS. Superação de dormência em sementes de três espécies de Parkia spp. Revista Brasileira de Sementes, v.33, n.3 p.533$542,2011$. 
Morais Júnior OP, Leão ÉF, Silva FCE, Silva DC, Aguiar JT, Peixoto N. Métodos para superação de dormência em sementes de murici. Revista Agrotecnologia, v.6, n.1, p.01, 2015.

Murakami DM, Bizão N, Vieira RD. Quebra de dormência de semente de murici. Revista Brasileira de Fruticultura, Jaboticabal - SP, v. 33, n. 4, p. 1257-1265, 2011.

Nascimento IL, Alves EU, Bruno RLA, Gonçalves EP, Colares PNQ, Medeiros MS. Superação da dormência em sementes de faveira (Parkia platycephala Benth). Revista Árvore, Viçosa-MG, v.33, n.1, p.35-45, 2009.

Oliveira LM, Bruno RLA, Silva KRG, Silva VDM, Ferrari CS, Silva GZ. Germinação e vigor de sementes de Sapindus saponaria L. submetidas a tratamentos pré-germinativos, temperaturas e substratos. Ciência Rural, v.42, p.638644, 2012.

Passos, I.R.S.; Matos, G.V.C.; Meletti, L.M.M.; Scott, M.D.S.; Bernacci, L.C.; Vieira, M.A.R. Utilização do ácido giberélico para a quebra de dormência de sementes de Passiflora nitida Kunth germinadas in vitro. Revista Brasileira de Fruticultura, Jaboticabal, v.26, n.2, p.380381, 2004.

Scalon, S.P.; Mussury, R.M.; Filho, H.S.; Francelino, C.S.F.; Florencio, D.K.A. Armazenamento e tratamentos pré-germinativos em sementes de jacarandá (Jacaranda cuspidifolia Mart.). Revista Árvore, Viçosa-MG, v.30, n.2, p.179185, 2006.

Scalon SPQ, Mussury RM, Wathier F, Gomes AM, Silva KA, Pierezan L, Filho HS. Armazenamento, germinação de sementes e crescimento inicial de mudas de Enterolobium contortisiliquum (Vell.) Morong. Acta Scientiarum Biological Sciences. Maringá, v.27, n.2, p.107-112, 2005.

Souza SM, Pires IE, Lima PCF. 1980a. Influência da embalagem e condições de armazenamento na longevidade de sementes florestais. p.15-24. In: Pesquisa Florestal no Nordeste Semi-árido: sementes e mudas. Boletim de Pesquisa n.2, EMBRAPA - CPTSA, Petrolina.

Souza SM, Pires IE, Lima PCF. 1980b. Efeito do tipo de embalagem e condições de armazenamento na preservação de sementes de aroeira (Astronium urundeuva) Engl. pp. 2530. In: Pesquisa Florestal no Nordeste Semi-árido: sementes e mudas. Boletim de Pesquisa n. 2, EMBRAPA CPTSA, Petrolina.

Vasconcelos Filho SC. Caracterização anatômica e histoquímica de folhas, calogênese e fitoquímica de calos de murici (Brysonima verbacifolia (L.) Ruch, ex Juss). 2008. 70p. Dissertação (Mestrado em Botânica) - Universidade Federal de Viçosa, Viçosa. 